\title{
Design and Testing a Remote Laser Listening System
}

\author{
Kasim M. Al-hity ${ }^{1}$, Albashir Zomrawi M.Yousif ${ }^{2}$
}

\begin{abstract}
Laser listening device is one the modern and powerful techniques in the field of remote sensing. In this research a simple system was developed to remote sensing the audio signals. The reflected laser spot position variation is dependent on the variation in the reflector position. A suitable receiver was designed for receiving and demodulating the audio signal. Light dependence resistance (LDR) was used in the receiver as an optical detector and it was tested in three cases. This system was tested to remote sensing the audio signals up to $5 \mathrm{~m}$ and it is easy to increase this distance. The results of this research are promising and one can conclude that this system can be used efficiently to sense the microvibrations, but with a careful alignment.

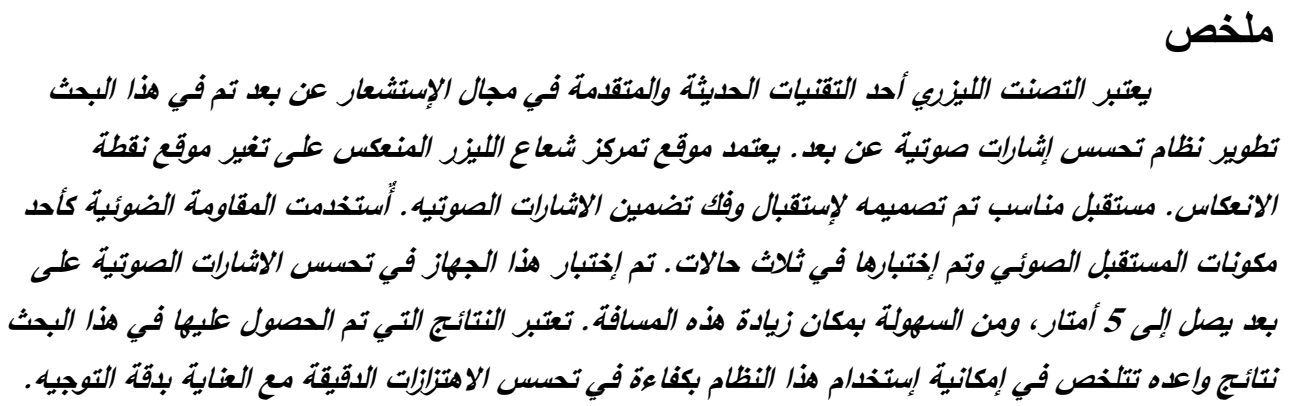

Key words: laser listener, vibration sensors, remote sensors.

\section{Introduction}

\footnotetext{
${ }^{1}$ Omdurman Islamic University, Faculty of Engineering Sciences.

${ }^{2}$ Karary University-Faculty of Engineering.
} 
The remote sensing of the micro vibration is made for a variety of reasons. But the major reason for performing a vibration test of a system is to determine its natural frequencies in hard environment. Another reason for vibration testing is to verify an analytical model proposed for the tested system. If the measured frequencies and mode shapes agree with those predicted by the analytical model, the model is verified and can be used in design and response prediction with some accuracy. Vibration testing is used to determine experimentally the dynamic durability of a particular device and also in machinery diagnostics for maintenance, by continuous monitoring of the natural frequencies of a structure [1].

The classical and common technique for vibration measurement is the piezoelectric accelerometer, which gives an electric signal proportional to the vibration acceleration. This signal can readily be amplified, analyzed, displayed, and recorded and so on. This dynamic device is usually enclosed in a case which is fastened to the surface whose vibration is to be measured. The body has a pointer fixed to it, which moves over a scale fastened to the case [2].

Seismic transducers, that convert mechanical motion into voltage, are mounted on a body whose vibrations are to be measured. As the vibrations occur, the seismic mass moves relative to the transducer housing, causing deformation in the piezoelectric crystal [3].

Vibration measuring devices having mass, spring, dashpot, etc. are known as seismic instruments. The quantities to be measured are displayed on a screen in the form of electric signal which can be readily amplified and recorded. The output of electric signal of the instrument will be proportional to the quantity which is to be measured. The input is reproduced as output very precisely. Similarly, other device known as an accelerometer is an instrument to measure the acceleration of a vibrating body. Vibrometer is designed with low natural frequency and accelerometer with high natural frequency. So vibrometer is known as low frequency transducer and accelerometer as high frequency transducer [4].

The subjective auditory sensation is a complex function of objective aspects of sound like frequency and intensity. Frequency of sound gives the subjective feeling of pitch whereas spectral distribution of energy gives subjective assessment of quality. Subjective loudness is a function of both frequency and intensity. 
The first component of the measurement system is the transducer - a microphone. This converts the signal into an electrical signal. Then we need what may be known as a signal conditioner to usually amplify the signal [5].

Vibration analysis has been conducted on large rotating machines for many decades. Proximity probes and accelerometers are traditionally used to measure bearing and shaft vibration in an effort to keep mechanical condition in check [6]. Optodyne vibration sensors are designed to detect the small increments of linear displacement that occur in vibrating structures or materials. These instruments are useful in a wide variety of applications, such .as hard disk assemblies, microelectronics processing and inspection equipment, material and component analysis and machine design [7].

The new Colibrys wide bandwidth capacitive accelerometers set new performance standards on vibration sensing and are expected to displace piezoresistive sensors in Health and Usage Monitoring Systems (HUMS), Aeronautics, Railway technologies and automotive crash testing applications. The recognized high performance level of capacitive sensors, well proven for inertial and seismic applications, is now also available for vibration sensing [8]. A finite element emulation of jet engine developed at Boeing Phantom Works is used to illustrate MISED. Four vibration sensors are placed at disperse locations on the engine subjected to imbalances at three locations. Each sensor measures the vibration spectrum in the $\mathrm{x}, \mathrm{y}$ and $\mathrm{z}$ directions [9]. The sensing technique described in this dissertation produces modal coordinates for monitoring and active control of structural vibration. The sensor array is constructed from strain-sensing segments. The segment outputs are transformed into modal coordinates by a sensor gain matrix [10]. Traditional magneto-electric vibration sensors and servo accelerometers have severe shortcomings when used to measure vibration where low frequency components predominate [11].

The properties of the micro vibration sensor device that it is a low cost device which make it available for any one to use and its small size assist it to be portable and easy to carry and manipulate. Its simple design makes the device to be constructed in any small lab. In addition to the properties, the utilization of laser beam in making measurement assist to measure micro vibration in remote fashion. Low power consumption is an addition advantage of this device. 


\section{Laser Listener Design}

In this system, the laser beam was send to reflecting vibrating body (side glass of car) and then the vibrations of the reflector position were modulated in the reflected laser beam, the reflection part of the beam is received by the receiver to measure the microvibration with the light dependent resistance detector (LDR) and converted to electric signal. The amplification of this signal then performed with the C1212C amplifier. Set of capacitors and resistors were arranged to filter the amplified signals to produce a fine signal.

Finally these signals are transferred to measurable values graphically using the vibrometer device. The block diagram of the microvibration sensor and the vibrating body, show Fig (1).

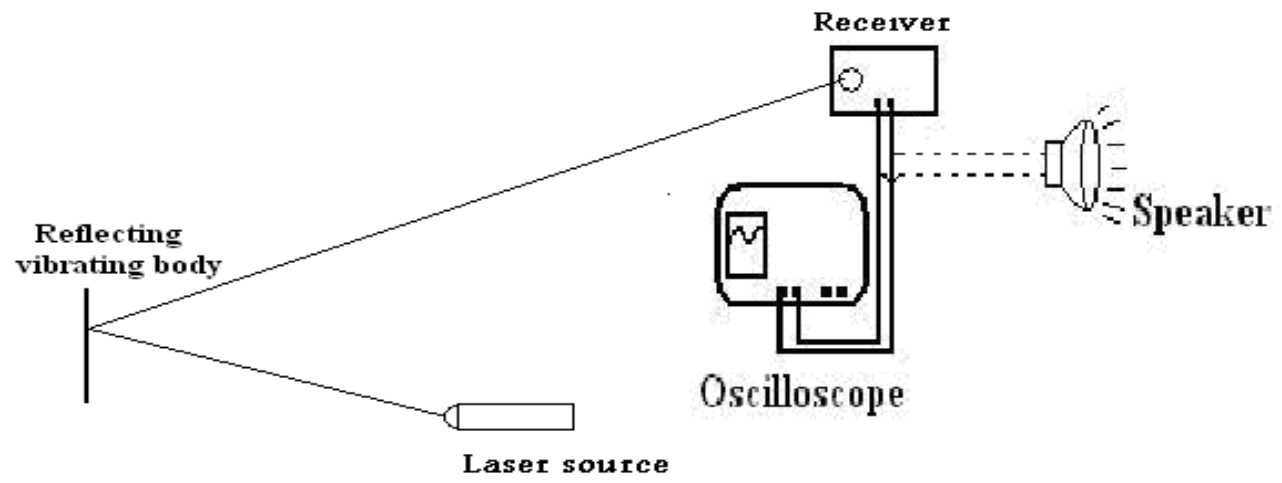

Figure (1): Block diagram of the laser listener system

The main three components of the sensor are: laser source, receiver and display unit.

Laser diode of $635 \mathrm{~nm}$, with output power less than $5 \mathrm{~mW}$ was used in this system to be as laser source. The receiver sensor consists of an electronic circuit with a light resistance detector (LDR) of 700nm sensitivity [12]. Figure (2) shows the developed receiver circuit of the laser listening system. 


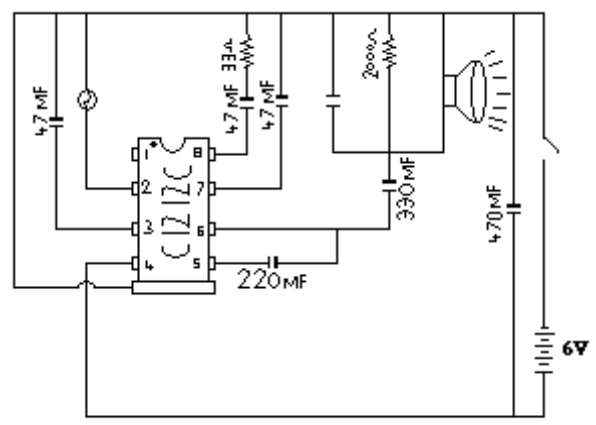

Figure (2): Block diagram of the receiver circuit.

An output device is required to transform the electric signals of the sensor to measurable value. An oscilloscope was used here to record the strength signal. Note that another output devices can be used such as speakers and graph meters.

\section{Results and Discussions}

The system was tested by sending the laser beam to the side glass of car and received the reflected part of it. Then compare this signal with the original signal of the radio speakers that put in the car. Three cases were recorded:

Case (1): very low outputs signal was illustrated in the figure (3). The measured signals (by remote sensing) of this laser listener is shown in figure (3-A), while the original signal of the radio speakers was shown in figure (3-B) in which the vibrator was put it in the car in a low volume.

Case (2): low outputs signal was illustrated in the figure (4). The measured signal was shown in figure (4-A) while figure (4-B) illustrated the original signals of the radio speakers in moderate volume.

Case (3): the same thing was illustrated in figure (5) but with high distortion outputs signal. 


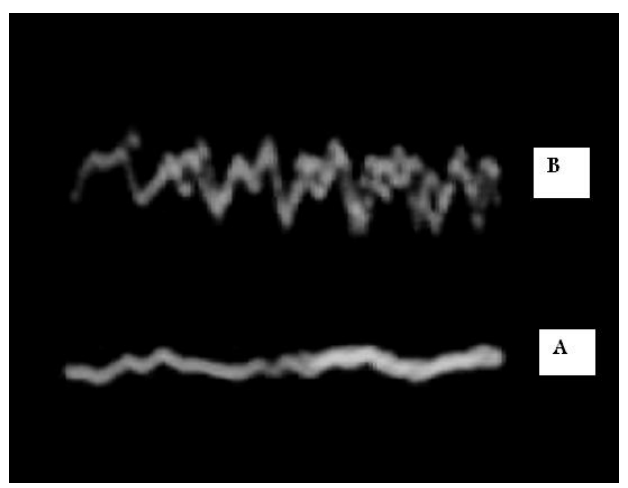

Figure (3): Original \& microvibration sensor signal at low volume, (A) Sensor signal \& (B) original signal.

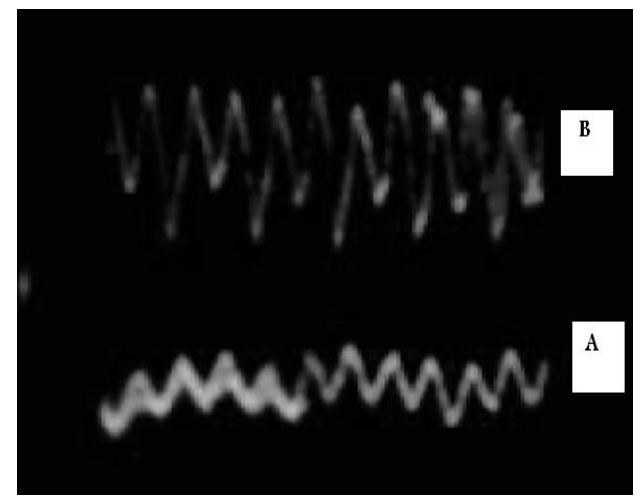

Figure (4): Original \& Micro Vibration Sensor signal at Mid Volume, (A) Sensor signal \& (B) Original Signal.

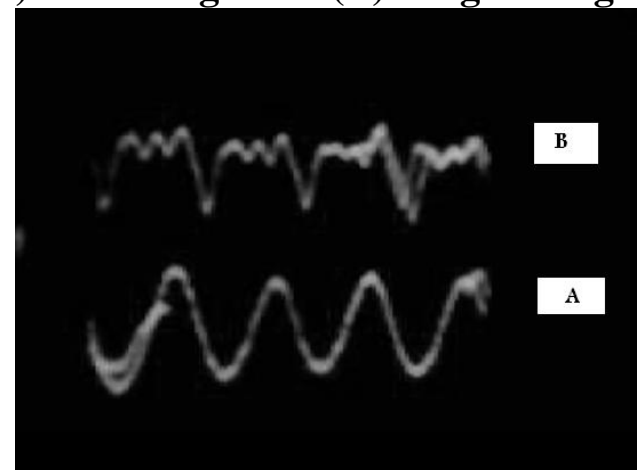

Figure (5): Original \& micro vibration sensor signal at high volume, (A) Sensor signal \& (B) original signal. 
Comparing graph (A) and graph (B) in figure (4), it can be found that the signals are convenient which implies that the sensor can efficiently measure the microvibration signal in the moderate volume.

\section{Conclusions}

By referring to the results obtained in this research, one can conclude that this sensor can efficiently sense the audio signals as well as the micromechanical vibrations. Variation of the output electrical signal of the photodetector depends on the overlapped area between the laser spot and the area of the photodetector as illustrated in figure (6).

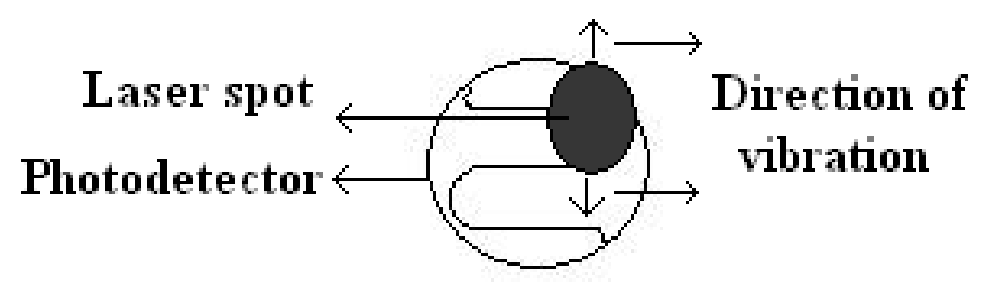

Figure (6): The overlapping between the laser spot and the photo detector.

\section{References}

1-Rao V. Dukkipati- J. Srinivas-Textbook of mechanical vibrations-March 2005.

2-C.F.Beards BSc, PhD, C Eng, MRAES, MFOA- Structural vibration: Analysis and damping -university of London - Britain 1996.

3-S.Graham Kelly-Fundamentals of mechanical vibrations - second edition 2000

4-V.P. Singh- Mechanical vibrations (Mechanical Engineering) (A Modern Approach) - 2005 .

5-Kewal Pujara-Vibrations and noise for engineers - 2004.

6-Mike Hoffer - Consumers Energy - Jackson, MI - USA, Andrew Tesla VibroSystM Inc. Montreal, QC - Stator Bar Vibration Sensors and FiberOptic Accelerometers - Canada 2004.

7-Via veneto, Bernaregio (MIILANO) Italy, WWW.optodyne.com Optodyne Laser Metrology 
8-New Sensor VS9000 will be presented at Sensor + Test NurembergColibrys extended bandwidth capacitive vibration sensors to displace piezoresistive technology for HUMS applications - 6-8 May 2008 - (booth 7-326) and at Sensors Expo Rosemont, IL, 9-11 June 2008 (booth 1113).

9-Sreeram Narayanan, John L. Vian, J.J. Choi, R.J. Marks, M.A. ElSharkawi, \& Benjamin B. Thompson- Missing Sensor Data Restoration For Vibration Sensors on a Jet Aircraft Engine $\square$ - (C2003 IEEE.

10-Hartono Sumali - Blacksburg, Virginia - A New Adaptive Array of Vibration Sensors - July 1997.

11-Yang Xueshan, Gao Feng and Hou Xingmin-Institute of Engineering Meehanics, China Earthquake Administration, Harbin 150080, Lowfrequency characteristics extension for vibration sensors - China - June 2004

12-Albashir Zomrawi Mohamed Yousif, M.Sc. thesis, Sudan University of science \& Technology, Institute of laser, 2009. 